\title{
Single walled carbon nanotubes for transport and delivery of biological cargos
}

\author{
Nadine Wong Shi Kam ${ }^{*}, 1$ and Hongjie Dai ${ }^{2}$ \\ ${ }^{1}$ Michigan Society of Fellows and Department of Chemical Engineering, University of Michigan, \\ Ann Arbor, Michigan, USA \\ ${ }^{2}$ Department of Chemistry, Stanford University, Stanford, California, USA
}

Received 17 July 2006, accepted 2 October 2006

Published online 25 October 2006

PACS 87.16.- b, 87.80.-y

The innate ability of carbon nanotubes to breach the cell membrane of various types of mammalian cells has been reported. Here, we present a summary of the various applications of carbon nanotubes as a cellular transport and delivery system for functional biological cargos. The internalization of SWNTs for transport and delivery into cells is mediated via endocytosis and does not appear to have any detrimental effect on either the transported cargo or the breached cell. The emergence of SWNT as a new class of cellular transporters holds many exciting promises for SWNT-based systems for drug delivery, protein delivery, gene therapy and cancer therapy applications.

C 2006 WILEY-VCH Verlag GmbH \& Co. KGaA, Weinheim

\section{Introduction}

The development of novel biomedical therapies is greatly dependent on the ability of drugs and other extracellular species to cross the cellular barrier [1]. As such for new strategies aimed at drug delivery and gene therapy applications to be deemed realizable, they often rely on the use of an agent of cellular transport. For cellular systems, this agent is often called a "transporter"; that is able to be loaded with a cargo of choice and have the ability to carry that cargo though the cellular membrane without any detrimental effect on its biological functionality. One such example is the use of viral vectors that possess membrane translocation properties to carry oligonucleotides and other cargos inside the cells. Other classes of transporters include liposomes, cell-penetrating peptides and other small molecules based on the sequence of HIV-Tat proteins [1-4]. These molecules are being deemed as the next generation of transporters in this new era of drug delivery and gene.

The convergence between nanotechnology and biology has over recent years garnered increasing interest. In particular, the development of novel applications and interfaces between nanomaterials and biological systems has been an active area of research. Carbon nanotubes have proven to be an excellent candidate for such applications, with efforts predominantly geared towards utilizing carbon nanotubes as a platform for biosensors [5-8]. Recently, we and others have reported that single-walled carbon nanotubes (SWNT) are capable of penetrating inside mammalian cells [9-18]. The cellular internalization of SWNT occurs without the need for any external transporter system. The nanoscale dimensions of SWNT, combined with their high aspect ratio, makes them amiable to high cargo loading, thus holding high promises for SWNT act as a new class of transport system.

\footnotetext{
" Corresponding author: e-mail: nwsk@umich.edu, Phone: +001 7347645512
} 
The development of nanotube-based cellular transport systems opens up new avenues for the potential application of the nanotubes to address current problems in the biological realm. This report provides an overview of the applications of SWNTs as transporters for proteins into mammalian cells and addresses critical questions, including: biocompatibility of the nanotubes material on the cellular level, cargo release ability upon internalization as well as the cellular fate of the transported cargo in terms of localization and biological functionality.

\section{Cellular transport mediated by carbon nanotubes}

Exploring the potential of carbon nanotubes for novel biological applications has been increasingly attractive more attention. In recent years, applications such as using carbon nanotubes as a platform for cellular growth and as a biosensor for protein systems have been reported $[5,19]$. In an effort to gain understanding on the interactions between SWNTs and biological species, we have studied the interfacing between single-walled carbon nanotubes and mammalian cell lines [12]. The carbon nanotubes were functionalized covalently by acid-treatment to afford dispersability in aqueous media. The presence of functional carboxyl groups following oxidation allowed subsequent strategies to either covalently or non-covalently link proteins to the nanotubes sidewalls.

Carbon nanotubes were fluorescently tagged and mixed with living mammalian cells at $37{ }^{\circ} \mathrm{C}$ and in a $5 \% \mathrm{CO}_{2}$ environment. Fluorescence was detected inside the cells following incubation with the labelled SWNTs, indicating internalization of carbon nanotubes inside mammalian cells. The observed ability of SWNTs to penetrate inside cells was further investigated by attaching proteins to the nanotubes' surface. A model protein system was initially investigated by relying on the biotin-streptavidin (SA) pair. Biotin is a small molecule that can be commercially obtained with an amine termination. The biotin moiety can subsequently be easily conjugated to the oxidized SWNT via an amide linkage, The biotin-streptavidin system is particularly convenient due to the highly specific interaction between biotin and streptavidin. As shown in Fig. 1a, after conjugation of the SWNT-biotin, the streptavidin can be attached to the conjugate via simple mixing of the SWNT conjugation with the protein solution. The internalization of the SWNT-biotin-SA (where the SA is fluorescently labelled) is verified by the fluorescence detected in the cells by confocal microscopy as shown in Fig. 1b.

Cellular transport mediated by SWNT transported has thus been shown for streptavidin via its highly specific interaction with biotin. In order to determine whether SWNT can act as a transported for generic proteins, the acid-treated, oxidized SWNTs were exposed to solutions of various proteins. Non specific binding of a variety of proteins onto the oxidized SWNT was observed and cellular internalization of the resulting SWNT-protein conjugates were internalized inside cells. The strong interaction between SWNT

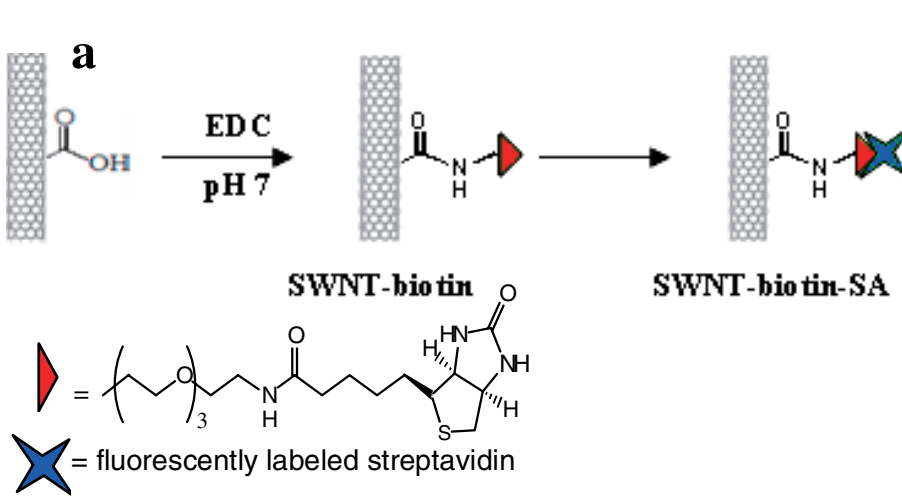

b

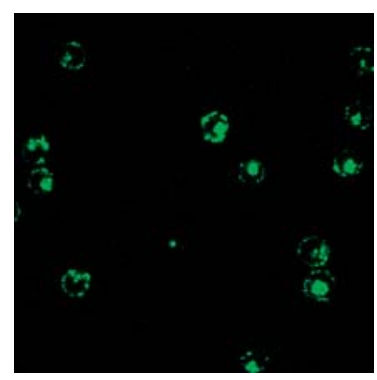

Fig. 1 (online colour at: www.pss-b.com) (a) Schematic of SWNT conjugates. (b) Confocal microscopy image of mammalian cells (HL-60) exposed to SWNT-biotin-SA conjugates. 
and proteins is attributed to attractive forces between the carboxyl groups on the SWNT surface and positively charged domains on the proteins. The non-specific, non-covalent attachment of proteins onto SWNTs thus enables a facile way to make SWNT as a generic transporter for proteins (MW $\leq 80 \mathrm{kD})$ [11]. The larger proteins are thought to have weaker interactions with SWNT due to the relatively small area of contact. The internalization of the nanotubes inside the cells did not markedly affect the cell viability and proliferation, therefore providing a promising indication of the biocompatibility of the nanotubes.

\section{Cellular delivery mediated by carbon nanotubes}

The internalization pathway for SWNT conjugates was confirmed to be endocytosis and specifically found to be mediated by clathrin receptors [10-13]. This endocytotic mechanism was found to be independent of the conjugation scheme of the nanotubes, since both covalently modified protein-SWNT conjugates or SWNT non-covalently coated with single stranded DNA appear to be internalized via endocytosis. Endocytosis is known as a general entry mechanism for various extra cellular materials and is energy-dependent uptake [20-22] hindered when incubations are carried out at low temperature $\left(4{ }^{\circ} \mathrm{C}\right.$ instead of $37^{\circ} \mathrm{C}$ ) or in ATP (adenosine triphosphate) depleted environments [21-23]. Cellular incubations carried out with SWNT conjugates at low temperatures or under conditions known to deplete cells from ATP along with standard incubations, indicated that the internalization was indeed hindered upon low-energy environments. Though the endocytosis mechanism differs from the one proposed by others $[14,24,25]$, it appears to confirm that our conjugates (individualized SWNTs or small bundles) penetrate inside the cells via an energy-dependent process, rather than by diffusion through the cellular membrane.

In order to fully assess the potential applications of SWNTs to the field of drug delivery and gene therapy, it is imperative to extend the ability of carbon nanotubes to transport biological cargos inside cells, to the ability to deliver the transported cargo, while preserving the latter's biological activity. In particular, the detachment of the drug or cargo species from the transporter molecule often needs to be realized such that the cargo can reach its target destination and still be biologically or chemically effective [3, 4, 26-29]. In order to achieve this goal, a non-covalent functionalization scheme is utilized that involves coating of the carbon nanotubes with a phospholipids-derived molecule as shown in Fig. 2.

The phospholipid molecule (PL) is of particular interest because of the co-existence of the hydrophobic backbone and hydrophilic tail (polyethylene glycol, PEG) and especially because of the presence of a terminal functional group that can be utilized for subsequent conjugation steps. The conjugation goal here was to introduce cleavable bonds between the SWNT and the biological cargo, such as a disulfide bond to enable release of the cargo from the SWNT transporter upon internalization Previous reports have shown that disulfide bonds can be broken under acidic $\mathrm{pH}$ environments and in particular in the lysosomal compartment of cells $[30,31]$. We have previously established that the SWNT conjugates are most likely localized into acidic endosomal vesicles due to the internalization mediated by endocytosis; the disulfide bonds are thus ideal for the SWNT transport systems. The disulfide bond was introduced by first modifying the SWNT with an amine terminated PL molecule, and relying on a heterobifunctional crosslinker (sulfo-LC-SPDP) to introduce a disulfide bond between the amine terminal of the PL and a

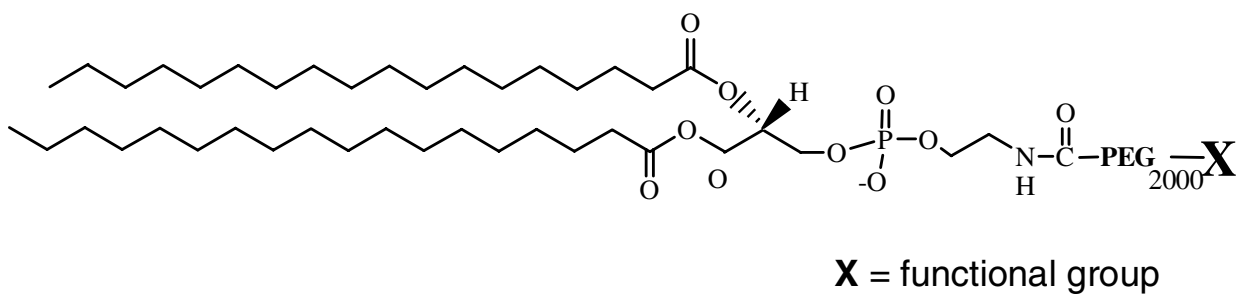

Fig. 2 Chemical structure of phospholipid molecule used for non-covalent functionalization of SWNT. 

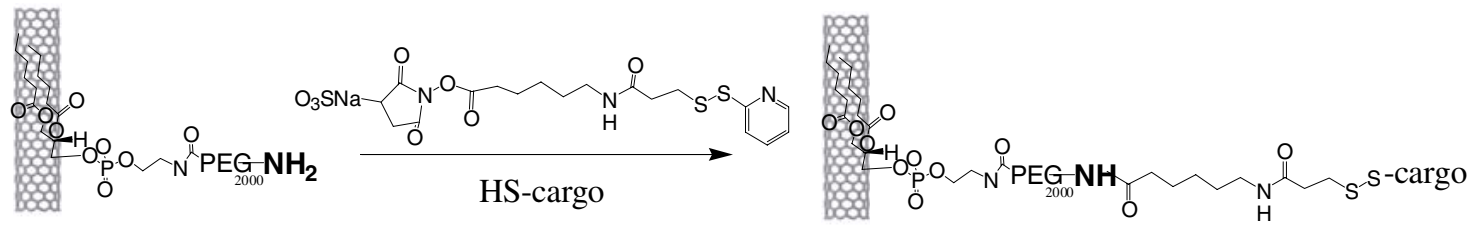

Fig. 3 Conjugation scheme to introduce disulfide bonds between the nanotube and the biological relevant cargo.

thiol-containing cargo molecule, as shown in Fig. 3. The presence of the disulfide bond was verified in a first place with by attaching a fluorescently DNA cargo to the nanotubes. In the presence of the disulfide bond, nuclear translocation of the DNA molecule is observed, thus indicating the detachment of the DNA cargo from the nanotube transporter (data not shown) [9].

Following the observation of DNA translocation, we aimed at achieving transport and delivery of more biologically relevant cargos via the SWNT transporter. Of particular interest is utilizing SWNT as a tool in the field of gene and protein therapy and in particular inRNA interference (RNAi). RNAi is a post-transcriptional gene silencing mechanism that is triggered via a process that degrades the messenger RNA, a mechanism responsible for protein production [32,33]. RNAi deemed highly promising for therapeutic knock-down of disease-causing genes as it is highly specific in nature. Though RNAi was initially discovered in plant cells, recently increasing interest has been devoted to applying this gene silencing technique to mammalian cells [34-39]. In mammalian cells, RNAi is induced by the introduction of short double-stranded RNA (or short interfering RNA, siRNA) into the cell cytoplasm. The siRNA needs to rely on a transporter to be introduced inside cells. For RNAi to be effected, release of the siRNA cargo is essential to prevent degradation inside the endosomal compartments as well as to enable the cargo to reach its destination inside the cytoplasm. To that effect, thiol-terminated siRNA was conjugated to SWNT with the inclusion of a disulfide bond.

A model system is set up in order to achieve silencing of a generic gene, lamin $\mathrm{A} / \mathrm{C}$ that is present in the nuclear lamina of a variety of cells [32]. SWNT-siRNA conjugates containing a disulfide bond was thus prepared with the siRNA sequence that is specific to lamin A/C. HeLa cells were used in these experiments since they are known to express the lamin $\mathrm{A} / \mathrm{C}$ protein. The cells were incubated for periods of up to $24 \mathrm{~h}$ and then fixed, stained with a primary antibody that is specific to lamin (anti-lamin), followed by incubation with a fluorescently labelled secondary antibody. In order to fully assess the RNAi effect mediated by the SWNT-siRNA, a commercially available transfection reagent, Lipofectamine [40], was also used. The protein expression of lamin $\mathrm{A} / \mathrm{C}$ was first established in untreated HeLa cells, by the
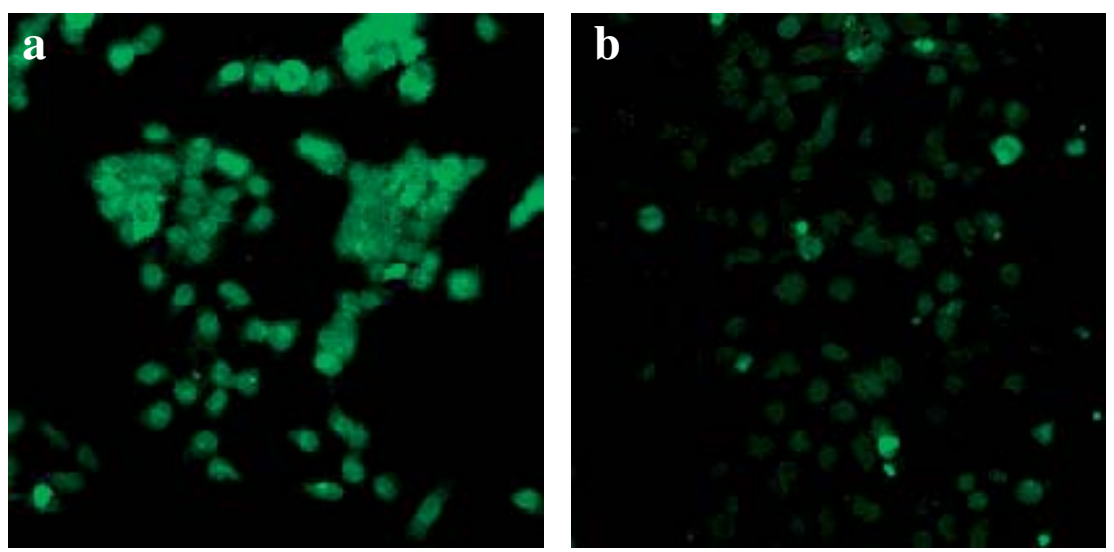

Fig. 4 (online colour at: www.pss-b.com) Lamin A/C silencing in HeLa cells. (a) Control, untreated HeLa cells after staining steps used to assess protein expression. (b) Protein expression in HeLa cells that were treated with SWNT-siRNA conjugates that contained a disulfide bond. 
staining steps with the primary and secondary antibodies, as shown in Fig. 4a. The presence of the lamin $\mathrm{A} / \mathrm{C}$ is detected by a high level of fluorescence from the cells.

The level of lamin A/C protein expression was assessed by confocal microscopy after the fluorescence staining process. As shown in Fig. 4b, a dramatic reduction in the fluorescence is detected in the stained cells, following treatment with the SWNT-siRNA conjugate. Furthermore, the SWNT-siRNA seemed to trigger silencing efficiency that was superior than the commercial transfection agent. It should be noted that incubation of cells with SWNT-siRNA conjugates that do not contain the disulfide bond showed inferior level of silencing (data not shown), thus indicating that the disulfide bond is necessary for cargo release. The higher efficiency can potentially be a result of high loading of the siRNA molecules onto the nanotube's surface, thus leading to a higher level of siRNA introduction inside the cells. This observation thus indicates that having a disulfide bond is essential for cargo delivery, and that the biological activity of the delivered cargo is preserved.

\section{Conclusion}

This study summarizes some of the recent work aimed at exploring the interactions between carbon nanotubes and biological systems. Both covalently and non-covalently functionalized carbon nanotubes have been shown to have the ability to be internalized inside mammalian cells. This ability has led SWNTs to be utilized as a novel class of transport system that mediate cellular internalization of biological cargos. The internalization of the carbon nanotubes is found to be mediated by the process of endocytosis. Determining the internalization mechanism was key to devising new strategies that allowed the nanotubes to not only carry the cargos inside the cells, but also have the ability to "release" these species. By utilizing a generic functionalization scheme that incorporate a disulfide bond between the SWNTs and a variety of molecules, ranging from simple fluorescent dyes to more complex species such as proteins, antibodies and oligonucleotides. The biological activity of the cargo is preserved following transport and delivery into the cell. This was demonstrated by effective gene silencing by siRNA introduced inside the cells via the nanotubes transporter. Current studies are under way to assess the applications of the SWNT-based transport and delivery system for other biological systems. In the context of the in-vitro studies summarized in this report, single-walled carbon nanotubes have been shown to lie at an exciting crossroads between biology and nanotechnology. The applications of carbon nanotubes in drug delivery, gene therapy and cancer therapies are emerging and need to be fully explored in a variety of systems, and eventually in in-vivo settings to fully establish their merits in the biomedical realm.

\section{References}

[1] C. M. Henry, Chem. Eng. News 82, 37 (2004).

[2] J. B. R. Lee, R. Wright, and P. A. Wender, Curr. Protein Peptide Sci. 4, 105 (2003).

[3] T. M. McAllen and P. R. Cullis, Science 303, 1818 (2004).

[4] D. A. Smith and H. vandeWaterbeemd, Curr. Opion. Chem. Biol. 3, 373 (1999).

[5] F. Balvavoine, P. Schultz, C. Richard, V. Mallouh, T. W. Ebbeson, and C. Mioskowski, Angew. Chem. Int. Ed. 38, 1912 (1999)

[6] K. Besteman, J. O. Lee, F. G. M. Wiertz, H. A. Heering, and C. Dekker, Nano Lett. 3, 727 (2003).

[7] R. J. Chen, S. Bangsaruntip, K. A. Drouvalakis, N. W. S. Kam, M. Shim, Y. M. Li, W. Kim, P. J. Utz, and H. J. Dai, Proc. Natl. Acad. Sci. USA 100, 4984 (2003).

[8] P. Qi, O. Vermesh, M. Grecu, A. Javey, Q. Wang, H. Dai, S. Peng, and K. J. Cho, Nano Lett. 3, 347 (2003).

[9] N. W. S. Kam, Z. Liu, and H. Dai, J. Am. Chem. Soc. 127, 12492 (2005).

[10] N. W. S. Kam, Z. Liu, and H. Dai, Angew. Chem. Int. Ed. 45, 577 (2006).

[11] N. W. S. Kam and H. Dai, J. Am. Chem. Soc. 127, 6021 (2005).

[12] N. W. S. Kam, T. C. Jessop, P. A. Wender, and H. Dai, J. Am. Chem. Soc. 126, 6850 (2004).

[13] N. W. S. Kam, M. O'Connell, J. A. Wisdom, and H. Dai, Proc. Natl. Acad. Sci. USA 102, 11600 (2005).

[14] A. Bianco, J. Hoebeke, S. Godefroy, O. Chaloin, D. Pantarotto, J.-P. Briand, S. Muller, M. Prato, and C. D. Partidos, J. Am. Chem. Soc. 127, 58 (2005).

[15] A. Bianco, K. Kostarelos, C. D. Partidos, and M. Prato, Chem. Commun. 5, 571 (2005). 
[16] P. Cherukuri, S. M. Bachilo, S. H. Litovsky, and R. B. Weisman, J. Am. Chem. Soc. 126, 15638 (2004)

[17] Y. Liu, D.-C. Wu, W.-D. Zhang, X. Jiang, C.-B. He, T. S. Chung, S. H. Goh, and K. W. Leong, Angew. Chem. Int. Ed. 44, 4782 (2005).

[18] Q. Lu, J. M. Moore, G. Huang, A. S. Mount, A. M. Rao, L. L. Larcom, and P. C. Ke, Nano Lett. 4, 2473 (2004).

[19] M. P. Mattson, R. C. Haddon, and A. M. Rao, J. Mol. Neurosci. 14, 175 (2000).

[20] M. Marsh and H. T. McMahon, Science 285, 215 (1999).

[21] S. Mukherjee, R. N. Ghosh, and F. R. Maxfield, Physiol. Rev. 77, 759 (1997).

[22] S. C. Silverstein, R. M. Steinman, and Z. A. Cohn, Annu. Rev. Biochem. 46, 669 (1977).

[23] S. L. Schmid and L. L. Carter, J. Cell Biol. 111, 2307 (1990).

[24] D. Pantarotto, J. Briand, M. Prato, and A. Bianco, Chem. Commun. 1, 16 (2004).

[25] D. Pantarotto, R. Singh, D. McCarthy, M. Erhardt, J. P. Briand, M. Prato, K. Kostarelos, and A. Bianco, Angew. Chem. 43, 5242 (2004)

[26] Y. W. Cho, J. D. Kim, and K. Park, J. Pharm. Pharmacol. 55, 721 (2003).

[27] T. B. Potocky, A. K. Menon, and S. H. Gellman, J. Biol. Chem. 278, 50188 (2003).

[28] P. Kolhe, E. Misra, R. M. Kannan, S. Kannan, and M. Lieh-Lai, Int. J. Pharmaceutics 259, 143 (2003).

[29] C. W. Lee, Macromol. Res. 12, 71 (2004).

[30] D. S. Collins, E. R. Unanue, and C. V. Harding, J. Immunology 147, 4054 (1991).

[31] B. Arunachalam, U. T. Phan, H. J. Geuze, and P. Cresswell, Proc. Natl. Acad. Sci. USA 97, 745 (1999).

[32] S. M. Elbashir, J. Harborth, W. Lendeckel, A. Yalcin, K. Weber, and T. Tuschi, Nature 411, 495 (2001).

[33] N. Agrawal, P. V. N. Dasaradhi, A. Mohmmed, P. Malhotra, R. K. Bhatnagar, and S. K. Mukherjee, Microbiol. Mol. Biol. Rev. 67, 657 (2003).

[34] N. J. Caplen, Gene Therapy 11, 1241 (2004).

[35] G. J. Hannon, Nature 418, 244 (2002).

[36] T. C. Karagiannis and A. El-Osta, Cancer Gene Therapy 12, 787 (2005).

[37] C. C. Mello and D. Conte, Nature 431, 338 (2004).

[38] M. Scherr, M. A. Morgan, and M. Eder, Curr. Medicinal Chem. 10, 245 (2003).

[39] S. M. Elbashir, J. Harborth, W. Lendeckel, A. Yalcin, K. Weber, and T. Tuschl, Nature 411, 494 (2001).

[40] V. Ciccarone, K. Schifferli, J. P. Pichet, K. Evans, L. Roy, P. Hawley-Nelson, and S. Bennett, FASEB Journal 14, A1473 (2000) 